\title{
Swim specialty affects energy cost and motor organization
}

\author{
L. Seifert ${ }^{1}$, J. Komar ${ }^{1}$, P. M. Leprêtre ${ }^{2}$, F. Lemaitre ${ }^{1}$, F. Chavallard ${ }^{1}$, M. Alberty ${ }^{3}$, \\ N. Houel ${ }^{4}$, C. Hausswirth ${ }^{5}$, D. Chollet ${ }^{6}$, P. Hellard ${ }^{4}$
}

\begin{abstract}
${ }^{1}$ Faculty of Sport Sciences, University of Rouen, Cetaps Upres EA3832, Mont Saint Aignan, France
${ }^{2}$ Faculté des Sciences du Sport, Université de Picardie Jules Verne, Laboratoire de Recherche Adaptations Physiologiques à l'Exercice et Réadaptation à l'Effort, EA3300, Amiens, France
\end{abstract}

${ }^{3}$ Laboratory of Human Movement Studies, Faculty of Sport Sciences, University of Lille, France

${ }^{4}$ Fédération Française de Natation, Département d'Etudes et Recherches, Paris, France

${ }^{5}$ INSEP, Biomechanics and Physiology, Paris, France

${ }^{6}$ Université de Rouen, CETAPS Laboratory EA3832, Mont Saint Aignan, France

Article publié dans : International Journal of Sports Medicine, 2010, vol. 31, $n^{\circ} 9$, pp. 624-630 (DOI: 10.1055/s-0030-1255066)

\begin{abstract}
The purpose of this study was to analyse the effect of swimmer specialty on energy cost and motor organization. The stroking parameters (velocity, stroke rate, stroke length, stroke index) and the index of coordination ( $\mathrm{IdC}$ ) of 6 elite sprinters were compared with those of 6 elite long-distance swimmers during an incremental swimming exercise test $(6 \times 300 \mathrm{~m}$ separated by $30 \mathrm{~s}$ of passive recovery) that progressively increased the energy cost. Energy cost (C), with its aerobic (Caero) and anaerobic (Canaero) components, was determined by measuring oxygen uptake (VO2) and blood lactate ([La]). Motor organization was assessed by analysis of video recordings from aerial and underwater side-view cameras. The results showed that throughout the test, both groups increased C, Canaero, stroke rate and IdC and decreased Caero and stroke length $($ all $\mathrm{P}<0.05)$. On the mean of the 300-m sets, sprinters had higher values for $\mathrm{C}(14.8$ vs. 12.9 $\mathrm{J} \bullet \mathrm{kg}-1 \cdot \mathrm{m}-1)$, Canaero (33.8 vs. $23.4 \%$ ), [La] (5.9 vs. $3.1 \mathrm{mmol} \bullet \mathrm{L}-1)$, stroke length (2.31 vs. $2.28 \mathrm{~m})$ and $\mathrm{IdC}(-11.2$ vs. $-21.7 \%)$ and lower values for Caero (66.2 vs. 79.6\%), VO2 net (2 825 vs. 2903 $\mathrm{mL} \cdot \mathrm{min}-1)$, stroke rate $(0.55 \mathrm{vs} .0 .62 \mathrm{~Hz})$ and stroke index $(2.96$ vs. $3.19 \mathrm{~m} 2 \bullet \mathrm{s}-1)$ than long-distance swimmers (all $\mathrm{P}<0.05$ ). For the same relative intensity, sprinters accumulated more lactate and swam more slowly than long-distance swimmers; they showed greater change in their arm coordination but their swimming economy was lower.
\end{abstract}

Key words: coordination - motor control - biomechanics - swimming - energy cost - specialty

\section{Introduction}

The energy cost of human locomotion differs greatly between land and water. Since the density of water is about 800 times that of air, aquatic locomotion requires much greater energy expenditure to overcome active drag. Di Prampero [13] compared several forms of locomotion at speeds corresponding to the world record for aerobic events of comparable duration and showed that the energy cost was highest in swimming $(20 \mathrm{~J} \bullet \mathrm{kg}-1 \cdot \mathrm{m}-1$ for $1500-\mathrm{m}$ free style at $1.67 \mathrm{~m} \bullet \mathrm{s}-1)$, being respectively 4.7 and 8.7 times greater than for running $5 \mathrm{~km}$ and cycling $10 \mathrm{~km}$. As the density of water is greater than air, the mechanical efficiency $(\eta \mathrm{M})$, which is the conversion of energy expenditure $\left(\mathrm{v}^{*}\right)$ (input) to mechanical work ( $\left.\mathrm{W}_{\text {tot }}\right)$ (output) (Equation 1), is low in swimming (8-12\%) [4][39][42][44]:

$\eta_{\mathrm{M}}=\mathrm{W}_{\text {tot }} / \mathrm{E}^{\cdot}$

The mechanical work (Wtot) is partitioned into the work done to overcome external forces (the external work, Wext) and the work done to accelerate and decelerate the limbs with respect to the center of mass (the internal work, Wint) [44]. The external work is related to the kinetic energy of the water and, because water does not allow for a solid push-off, Wext is composed of the work needed to overcome drag (Wd) 
that contributes to propulsion and the work wasted in the water $(\mathrm{Wk})$. The relationship between $\mathrm{Wd}$ and Wk is defined as the Froude efficiency $(\eta \mathrm{F})$ (Equation 2) [44]:

$\eta_{\mathrm{F}}=\mathrm{W}_{\mathrm{d}} / \mathrm{W}_{\mathrm{d}}+\mathrm{W}_{\mathrm{k}}(2)$

Finally, the propelling efficiency $(\eta \mathrm{P})$ is $[39][44]$ :

$\eta_{\mathrm{P}}=\mathrm{W}_{\mathrm{d}} / \mathrm{W}_{\text {tot }}(3)$

Thus, the combination of Equations 1 and 3 (in Equation 4) highlights the importance of propelling efficiency and the work used to overcome drag forces as factors determining energy expenditure and the energy cost in swimming:

$\mathrm{E}^{\cdot}=\mathrm{Wd} /(\eta \mathrm{P} \cdot \eta \mathrm{M})(4)$

Toussaint et al. [39] showed that $\eta \mathrm{P}$ varies between $46 \%$ and $77 \%$ for top-level swimmers. Moreover, Toussaint [38] observed lower $\eta \mathrm{P}$ for triathletes (44\%) than for competitive swimmers (61\%). Cappaert et al. [7] reported differences in $\eta \mathrm{P}$ for sprint (48\%), middle-distance (56\%) and long-distance swimmers $(62 \%)$, whereas $\eta \mathrm{M}$ did not change within groups, being close to $4-5 \%$.

Therefore, the swimmer specialty (sprint vs. long-distance) and the type of training, both of which are related to different organismic characteristics (muscular, anthropometric, metabolic and cardiorespiratory) $[3][6][11][12]$, may influence propelling efficiency in a task-dependent manner [7][38][41]. Sprinters, for example, are characterized by greater maximal strength and higher glycolysis and ATP-PCr enzymatic activity than long-distance swimmers [12][24]. Costill et al. [11] showed that the contribution of muscular strength to $25-\mathrm{m}$ and $400-\mathrm{m}$ swimming performance dropped to $86 \%$ and $58 \%$, respectively, suggesting that strength capacity contributes more to sprint performance. In fact, for middle- and long-distance events, the swimming speed at the lactate threshold and VO2 max were the most important factors for high-level performances [11][12]. Thus, according to Equation 4, the results of Cappaert et al. [7] suggested that the higher $v^{*}$ of the sprinters may have been due to their higher $\mathrm{Wd}$ and lower $\eta \mathrm{P}$.

However, the forward body displacement and thus the energy cost during aquatic locomotion are not only related to the Froude and propelling efficiencies, but also depend on the timing between the actions responsible for the external work, namely inter-limb coordination. Chollet et al. [10] assessed inter-arm coordination in front crawl, using an index of coordination (IdC) which quantified the time gap between 2 propulsive phases. These authors emphasized that another way to achieve efficient motor organization is to maintain a high average swimming speed by minimizing the time gap between 2 propulsive phases [10]. Propulsive discontinuities are among the factors (such as fluctuation of force impulse, active drag, etc.) that cause large intra-cyclic speed variations [29][34], thereby increasing the energy cost [5]. Hence, the opposition mode of inter-arm coordination, meaning the absence of a lag time between arm propulsions (i. e., perfect propulsive continuity), seems the most economic [8]. From this perspective, catch-up coordination could appear less economic as a lag time occurs between the propulsions of the left and right arms [10] and could lead to intra-cyclic speed variations. In fact, there is not an "ideal" coordination mode because coordination depends on the relations between interacting constraints (task, environment and organismic [33][36]). For example, at high speed (above $1.8 \mathrm{~m} \bullet \mathrm{s}-1$ ), where environmental constraints are elevated due to high active drag, superposition coordination mode (i. e., partial overlap of the 2 arm propulsions) appears to be the only effective and economic choice [33]. Organismic characteristics also influence arm coordination, as demonstrated for gender [30] and physical impairments and disabilities [28][34]. Several comparative studies have shown that only elite sprint swimmers reach high swimming speeds and switch to the superposition coordination mode [18][33], whereas less skilled swimmers [33] and triathletes [18] remain in catch-up mode. For the latter, this result could be explained by the high proportion of training in aerobic endurance [18] where speed is less than $1.8 \mathrm{~m} \bullet \mathrm{s}-1$. One case study highlighted that IdC increased when swim speed and energy cost increased [23]. Thus, when speed and energy cost increase, it is expected that swimmers will adapt their inter-arm coordination to follow the imposed pacing and to keep their propulsion as economic as possible. Given that sprint swimmers usually swim at speeds requiring greater anaerobic energy expenditure than long-distance swimmers [6], the aim of this study was to analyse the effect of swimmer specialty on energy cost and motor organization (arm coordination and stroking parameters).

\section{Material and Methods}

\section{Participants}


12 elite male front crawl swimmers voluntarily participated in this study in 2007 and composed 2 groups: 6 specialists in long distance (from 5 to $25 \mathrm{~km}$ ) and 6 specialists in sprint $(50 \mathrm{~m}$ and $100 \mathrm{~m}$ ). The longdistance swimmer characteristics were: $20.7 \pm 5.7$ years, $176 \pm 5 \mathrm{~cm}, 67.3 \pm 8.7 \mathrm{~kg}$. This group included 4 swimmers on the national junior team, 2 international finalists in the World Cup, and a European champion of the $10-\mathrm{km}$. The times in the $400-\mathrm{m}$ and $1500-\mathrm{m}$ events for this group were $243.70 \pm 4.63 \mathrm{~s}$ and $954.64 \pm 15.03 \mathrm{~s}$, respectively. The sprinter characteristics were: $22.2 \pm 4.4$ years, $183 \pm 7 \mathrm{~cm}, 73.3 \pm 9.5 \mathrm{~kg}$. The times in the 50-m and 100-m events for this group were $22.97 \pm 0.59 \mathrm{~s}$ and $51.86 \pm 1.68 \mathrm{~s}$, respectively. The protocol was approved by the University ethics committee and explained to the swimmers, who then gave their written consent to participate. This study was conducted in accordance with recognized ethical standards and the national/international laws reported by Harriss and Atkinson [17].

\section{Swim trials}

In a 50-m open pool $\left(26^{\circ} \mathrm{C}\right)$, the swimmers performed an incremental test. The test consisted of 6 consecutive $300-\mathrm{m}$ trials separated by 30 -s resting intervals. The starts were performed from the wall of the pool and, because they were breathing through a snorkel, the swimmers were asked to do hand turns rather than flip turns. Individual personal-best 400-m freestyle performances recorded within the month preceding the testing period were used to determine the paces of the incremental sets. The pace of the first $300 \mathrm{~m}$ was $30 \mathrm{~s}$ slower than the time required to swim $300 \mathrm{~m}$ at the adjusted $400-\mathrm{m}$ race pace. This time was then reduced by $5 \mathrm{~s}$ for each consecutive $300 \mathrm{~m}$ until the final $300 \mathrm{~m}$. Swimmers were verbally encouraged to reach their maximal speed during the final $300 \mathrm{~m}$. Thus, each set of $300 \mathrm{~m}$ represented the same relative intensity for both sprint and long-distance swimmers. During a pilot study, the amount of time lost because of snorkel use $(0.58 \mathrm{~s}$ per $50 \mathrm{~m})$ was determined for each swimmer by comparing 2 conditions, with and without the snorkel, during a 50-m trial at maximal speed. The time lost from executing hand turns instead of conventional flip turns (1.18 s per turn) was determined for each swimmer by comparing 2 conditions, flip turn without snorkel and hand turn with snorkel, at maximal speed. They were thus taken into consideration to adjust the speed of the sets. Swimming speed was monitored with an Aquapacer 'Solo' (Challenge and Response, Inverurie, UK). Markers were positioned every $2.5 \mathrm{~m}$ along the edge of the pool, and an operator then walked along the deck with a stick immerged in the water to indicate the prescribed swim speed to the swimmer. As done in previous studies, the operator used the auditory signal of the Aquapacer in correspondence with the visual markers on the deck to match his walking pace [2]. The swimmers were asked to maintain speed by keeping their heads at the level of the immerged stick of the operator with a range $<1 \mathrm{~m}$.

\section{Video recording and stroking parameters}

Aerial and underwater $(0.5 \mathrm{~m})$ side-view cameras (Panasonic NV-GS17, $50 \mathrm{~Hz}$ ) were superposed and fixed on the right side of the pool. These cameras video-taped 2 strokes taken in the central part of the pool. A video timer was included in the underwater view; this view was then synchronized and genlocked to the aerial view with Adobe Premiere (C). A calibration frame of $5 \mathrm{~m}$ in the horizontal axis and $2 \mathrm{~m}$ in the vertical axis was positioned on the floor of the pool, orthogonally to the external side-view camera, for measuring time over a 5-m distance to obtain the swim speed $(\mathrm{v}$, in $\mathrm{m} \bullet \mathrm{s}-1)$. When the front of the swimmer's head reached the edge of the frame and left the second edge of the frame, time was recorded. The arm stroke rate ( $\mathrm{SR}$, in $\mathrm{Hz}$ ) was calculated from hand entry at the first stroke to hand entry at the second stroke. The stroke length (SL, in $\mathrm{m} \bullet$ stroke-1) was calculated from the average speed (v) and the arm stroke rate (SR):

$\mathrm{SL}=\mathrm{v} \cdot \mathrm{SR}(5)$

According to Costill et al. [11], the stroke index (SI, in $\left.\mathrm{m} 2 \bullet \mathrm{s}^{-1}\right)$ is an indicator of swim efficiency and relates to the product of swimming speed $(\mathrm{v})$ and stroke length $(\mathrm{SL})$ :

$\mathrm{SI}=\mathrm{V} \cdot \mathrm{SL}(6)$

\section{Arm coordination}

Arm movement was broken down into 4 phases (entry, pull, push and recovery) [10]. The absolute duration of each phase was measured with a precision of $0.02 \mathrm{~s}$ from the 2 synchronized side-views, and then the relative duration of each phase was expressed as a percentage of complete stroke duration. The duration of the propulsive phase is the sum of the pull and push phases, and the duration of the non-propulsive phase is the sum of the entry and recovery phases. Arm coordination was quantified using the index of coordination (IdC) [10]. When a lag time occurred between the propulsive phases of the 2 arms, the stroke coordination was called 'catch-up' (IdC $<0 \%)$. When the propulsive phase of one arm started when that of the other arm ended, the coordination was called 'opposition' ( $\mathrm{IdC}=0 \%$ ). When the propulsive phases of the 2 arms 
overlapped, the coordination was called 'superposition' ( $\mathrm{IdC}>0 \%$ ). The $\mathrm{IdC}$ was calculated for 2 strokes per $50 \mathrm{~m}$ taken in the 10-m central part, then averaged for the 3 laps of $50 \mathrm{~m}$ composing the last $150 \mathrm{~m}$, to correspond to the analysis of the oxygen uptake. The IdC was expressed as a percentage of complete arm stroke duration.

\section{Energy cost}

During exercise, minute ventilation $\left(\mathrm{v}^{\cdot} \mathrm{v}^{*} \mathrm{E}\right)$, oxygen uptake $\left(\mathrm{v}^{\cdot} \mathrm{O} 2\right)$ and carbon dioxide production $\left(\mathrm{v}^{*} \mathrm{CO} 2\right)$ were recorded breath-by-breath by the K4b2 telemetric gas exchange system (Cosmed, Roma, Italy) [16][27], which was calibrated according to the manufacturer's instructions before each test. Ectopic artefacts were manually eliminated and data were then averaged every $5 \mathrm{~s}$. A capillary blood sample was obtained from the finger no more than $30 \mathrm{~s}$ after the end of the first 5 sets and 3 min after the last set and analysed for blood lactate concentration (Lactate Pro LT, Arkay Inc., Kyoto, Japan) [25]. The samples were also taken at rest and at the end of each $300 \mathrm{~m}$ during the all-out exercise. The energy cost per unit distance $(\mathrm{C}, \mathrm{mLO} 2 \cdot \mathrm{kg}-1 \cdot \mathrm{m}-1)$ was defined as:

\section{$\mathrm{C}=\mathrm{E} \cdot \mathrm{v}(7)$}

where $\mathrm{E}^{\cdot}$ is the total metabolic energy expenditure (aerobic and anaerobic pathways) expressed in $\mathrm{mLO} 2 \cdot \mathrm{min}-1 \cdot \mathrm{kg}-1$ and $\mathrm{v}$, in $\mathrm{m} \bullet \mathrm{min}-1$, is the swimming speed [13][15] at sub-maximal and maximal intensities. The aerobic component of swimming energy cost (Caero) was equal to the ratio between VO2net (i. e., the difference between the VO2 measured during the last minute of each swimming stage and its value at rest) and the swimming speed [13][15]. Caero was calculated from the steady state of $v^{*} \mathrm{O} 2$ [13][15], which corresponded to the last $150 \mathrm{~m}$ composing the $300 \mathrm{~m}$. The net energy cost of anaerobic glycolysis (Canaero) was estimated from blood lactate. Blood lactate measures ( $\mathrm{mmol}$ ) were converted to oxygen equivalent values as $3 \mathrm{mLO} 2 \cdot \mathrm{kg}-1$ of bodyweight per mmol of blood lactate [14]. Thus, C calculated as the addition of Caero and Canaero represented the energy expended to cover one unit of distance while swimming at a given speed and with a given stroke (anaerobic alactic energy sources seem to be neglected, or are assumed to be reduced, when evaluating $\mathrm{v}^{*}$ for $200-\mathrm{m}$ or longer events [26]). Finally, $\mathrm{C}$ is given in $\mathrm{J} \cdot \mathrm{Kg}-1 \cdot \mathrm{m}-1$, assuming that $1 \mathrm{mLO} 2$ consumed by the human body yields $20.9 \mathrm{~J}$ (which is true for a respiratory ratio of 0.96$)$ [6][13].

\section{Statistical analysis}

All values are given as mean \pm SD. A normal distribution (Ryan Joiner test) and the homogeneity of variance (Bartlett test) and authorized parametric statistics (Minitab 15.1.0.0, Minitab Inc., 2006) were verified. 3-way ANOVA (set $\times$ group $\times$ subject) analysed the effects of the incremental tests on the physiological and stroking parameters and IdC comparing the 2 groups (fixed factor: set and group; random factor: subject; $n=6$ sets $\times 2$ groups $\times 6$ subjects $=72$ ). The Pearson correlation test and linear regression then studied the relationships between energy cost (C, Caero, Canaero) and the IdC for each subject. Last, the values of the slope (modelling the linear regression) of the sprinters' group were compared with those of the long-distance swimmers' group with the Kruskal-Wallis test. For all tests, the level of significance was fixed at $\mathrm{P}<0.05$.

\section{Results}

\section{Swim set effect}

From the first to the last $300 \mathrm{~m}$, all subjects increased speed $(\mathrm{F} 5,52=33.2, \mathrm{P}<0.001)$ by increasing the stroke rate $(\mathrm{F} 5,52=46.7, \mathrm{P}<0.001)$ and decreasing the stroke length $(\mathrm{F} 5,52=15.9, \mathrm{P}<0.001)([$ Fig. 1]). For both groups ([Table 1] ), a significant increase in energy cost $(\mathrm{C})$ was observed $(\mathrm{F} 5,52=55.1, \mathrm{P}<0.001)$ and Caero decreased whereas Canaero increased (F5,52 = 9.5, $\mathrm{P}<0.001)$; however, the aerobic pathway remained the main contributor to $\mathrm{C}(>60 \%)$. The increase in $\mathrm{C}$ was related to a significant increase in VO2net $(\mathrm{F} 5,52=21.6, \mathrm{P}<0.001)$ and blood lactate concentration $(\mathrm{F} 5,52=47.6, \mathrm{P}<0.001)$. The $\mathrm{IdC}$ increased for both groups $(\mathrm{F} 5,52=10.3, \mathrm{P}<0.001)$ over the 6 sets of $300 \mathrm{~m}$, but it remained negative, meaning that the arm coordination was in catch-up mode ([Fig. 2]).

\section{Group effect}

On the mean of the 6 sets of $300 \mathrm{~m}$, the long-distance swimmers showed a higher mean speed $(1.39 \pm 0.10$ vs. $1.27 \pm 0.09 \mathrm{~m} \bullet \mathrm{s}-1 ; \mathrm{F} 1,52=62.4, \mathrm{P}<0.001)$, stroke rate $(0.62 \pm 0.07$ vs. $0.55 \pm 0.04 \mathrm{~Hz} ; \mathrm{F} 1,52=10.5$, $\mathrm{P}=0.02)$ and stroke index $(3.19 \pm 0.38$ vs. $2.96 \pm 0.32 \mathrm{~m} 2 \cdot \mathrm{S}-1 ; \mathrm{F} 1,52=43.7, \mathrm{P}<0.001)$ and a lower stroke 
length $(2.28 \pm 0.21$ vs. $2.31 \pm 0.15 \mathrm{~m} \cdot$ stroke $-1 ; \mathrm{F} 1,52=8.3, \mathrm{P}=0.006)$ and $\mathrm{IdC}(-21.7 \pm 2.3$ vs. $-11.2 \pm 3.6 \%$; $\mathrm{F} 1,52=561.8, \mathrm{P}<0.001)$ than the sprint swimmers ([Fig. 1]). On the mean of the 6 sets, the long-distance swimmers expended lower $\mathrm{C}(\mathrm{F} 1,52=13.9, \mathrm{P}<0.001)$, lower Canaero $(\mathrm{F} 1,52=15.3, \mathrm{P}<0.001)$ and higher Caero $(\mathrm{F} 1,52=15.3, \mathrm{P}<0.001)$ than the sprint swimmers ([Table 1]). The long-distance swimmers also had lower blood lactate concentration $(3.1 \pm 2.7$ vs. $5.9 \pm 2.2 \mathrm{mmol} \cdot \mathrm{L}-1)(\mathrm{F} 1,52=10.4, \mathrm{P}=0.02)$ and greater $\mathrm{VO} 2$ net $(2903 \pm 641$ vs. $2825 \pm 691 \mathrm{~mL} \cdot \mathrm{min}-1)(\mathrm{F} 1,52=6.2, \mathrm{P}=0.048)$ than the sprinters on the mean of the 6 sets.

The Pearson test of correlation showed positive relationships between $\mathrm{IdC}$ and $\mathrm{C}$ for the sprint (individual correlation coefficients ranged from $0.86<\mathrm{r}<0.98$, all $\mathrm{P}<0.05, \mathrm{n}=6$ ) and long-distance swimmers (individual correlation coefficients ranged from $0.81<\mathrm{r}<0.92$, all $\mathrm{P}<0.05, \mathrm{n}=6$ ). [Fig. 2] indicates the linear regression between $\mathrm{IdC}$ and $\mathrm{C}$ for the sprint (individual regression coefficients ranged from $0.75<$ $\mathrm{R} 2<0.96$, all $\mathrm{P}<0.05, \mathrm{n}=6$ ) and long-distance swimmers (individual regression coefficients ranged from $0.66<\mathrm{R} 2<0.85$, all $\mathrm{P}<0.05, \mathrm{n}=6$ ), and [Fig. 3] indicates the linear regression between IdC and Caero, and between IdC and Canaero, for the sprint (individual regression coefficients ranged from $0.67<\mathrm{R} 2<0.96$, all $\mathrm{P}<0.05, \mathrm{n}=6$ ) and long-distance swimmers (individual regression coefficients ranged from $0.66<\mathrm{R} 2$ $<0.92$, all $\mathrm{P}<0.05, \mathrm{n}=6$ ). The Kruskal-Wallis test showed a significantly higher slope for the sprint swimmers in comparison with the long-distance swimmers, concerning the linear regression between IdC and $\mathrm{C}$, between $\mathrm{IdC}$ and Caero, and between $\mathrm{IdC}$ and Canaero (all $\mathrm{P}<0.05$ ).

\section{Discussion}

The main finding of our study was that the progressive $6 \times 300-\mathrm{m}$ test elicited a predominantly aerobic state that increased energy cost and Canaero, which was more pronounced in the sprinters than in the longdistance swimmers. Sprinters (i) train more using the anaerobic pathway and (ii) are accustomed to a wider range of speeds and are able to reach higher maximal speeds. This aerobic test may have caused earlier muscular fatigue in these swimmers, who had lower performances (swim speed), lower efficiency (stroke index) and greater changes in arm coordination (increase in IdC) throughout the test than the long-distance swimmers.

\section{Energy cost and stroking parameters}

Our $\mathrm{C}$ values were in accordance with the literature, notably with the results of Zamparo et al. [43], who reported that elite long-distance swimmers had $\mathrm{C}$ of $15.4 \mathrm{~J} \bullet \mathrm{kg}-1 \bullet \mathrm{m}-1$ for a speed of $1.43 \mathrm{~m} \bullet \mathrm{s}-1$ assessed during a $2-\mathrm{km}$ trial. For our entire population, stroke rate increased while stroke length decreased when speed and $\mathrm{C}$ increased. This finding also agreed with the findings of Zamparo et al. [43], who compared a test of $3 \times 400 \mathrm{~m}$ with progressive speed pre- and post-2-km trial (swum at the speed of $10 \mathrm{~km}$ ). These authors showed that fatigue in elite long-distance swimmers led to an increase in stroke rate and a decrease in stroke length. Barbosa et al. [4] observed similar changes in the stroking parameters with fatigue during a progressive test of $7 \times 200 \mathrm{~m}$. The authors modelled these changes, showing significant quadratic relationships between speed and stroke rate $(R 2=0.82)$ and between speed and stroke length $(R 2=0.65)$ and linear relationships between energy cost and stroke rate $(\mathrm{R} 2=0.20)$. Our results also indicated a higher mean $\mathrm{C}$ in the sprinters than in the long-distance swimmers, which was in accordance with Chatard et al. [9], who observed a C $15.7-16.5 \%$ greater for sprinters. In running, $\mathrm{C}$ was also about $10 \%$ greater in sprinters than in long-distance runners [19].

\section{Aerobic and anaerobic components of $\mathrm{C}$}

According to Capelli et al. [6], the increase in C corresponded to a decrease in Caero and an increase in Canaero (which are expressed in \% of $\mathrm{C}$ ) in both our groups. However, our long-distance swimmers expended greater Caero for a higher swimming speed than the sprint swimmers, meaning that long-distance swimmers might be capable of reaching a higher percentage of maximal speed at the 400-m race pace (i. e., a higher percentage of maximal aerobic speed, taking into account that Lavoie and Montpetit [20] showed that the speed of the 400-m swum at maximal intensity is highly correlated with maximal aerobic speed) with lower blood lactate accumulation. This is in accordance with the results of Shepard et al. [35], who showed higher blood lactate concentration and lower oxygen expenditure for sprinters vs. medium- and long-distance swimmers during maximum exercise. Similarly, Olbrecht et al. [24] demonstrated that for the same blood lactate concentration, shorter exercise times resulted in higher mean anaerobic energy delivery, but that better aerobic endurance also increased the anaerobic contribution. This may explain why in our experiment the long-distance swimmers had lower $\mathrm{C}$ and produced lower blood lactate concentration than sprinters: our protocol imposed swim trials in a predominantly aerobic state and long-distance swimmers are used to this physiological task. Conversely, sprinters use the anaerobic energy system more during training and racing, and glycolysis and ATP-PCr enzymatic activity have a lower energy delivery rate than the aerobic energy system [12][20][24]. It could thus be hypothesized that the sprinters became fatigued 
more quickly during our aerobic tests and thus had lower swim economy and efficiency. Although they had greater stroke lengths than the long-distance swimmers, these latter had lower energy cost (C, Caero), higher efficiency (stroke index) and higher performance (swim speed). This hypothesis was confirmed by Cappaert et al. [7], who showed that aerobic swims show greater propelling efficiency $(\eta p=43.5 \%)$ than anaerobic swims $(\eta p=33.4 \%)$. Cappaert et al. [7] also reported higher $\eta p$ for long-distance swimmers $(62 \%)$ than for sprinters $(48 \%)$.

\section{Arm coordination}

The index of coordination (IdC) increased for both groups, suggesting that increases in speed and fatigue led to changes in arm coordination (according to [23]) associated with changes in stroking parameters (as previously shown). However, the IdC of the long-distance swimmers was very negative for all sets of 300 $\mathrm{m}$ (mean IdC $<-20 \%)$, showing greater catch-up coordination mode than in the sprinters $(-15 \%<\mathrm{IdC}$ $<-5 \%)$. Moreover, the slopes of the linear regressions IdC - C, IdC - Caero and IdC - Canaero were significantly steeper for the sprinters ([Fig. 2], [3]), suggesting they changed their arm coordination more than the long-distance swimmers. Thus, a question arose: Why did the long-distance swimmers reach higher speed at each set, displaying greater efficiency (higher stroke index) and economy (lower C) than the sprinters, even though the latter changed their arm coordination and relied less on catch-up mode?

First, as previously suggested, it could be hypothesized that the sprint swimmers modified their arm coordination more than the long-distance swimmers because they were more trained in anaerobic exercise; they thus exhibited earlier muscular fatigue during a progressive swimming test that essentially imposed aerobic swims. Conversely, the long-distance swimmers, who were more trained in aerobic exercise, kept their coordination in great catch-up because they were highly focused on their hydrodynamic position in order to minimize resistive forces and maximize efficiency [21].

Indeed, several studies [1][32] concerning sprint exercise have shown that IdC increases with fatigue and, notably, the relative duration of the propulsive phases (pull and push) increases as the swimmers slow their hand speed [37] and apply lower force and power output [40]. Alberty et al. [1] reported an increase in IdC from -6.5 to $-3.3 \%$ during a $4 \times 50 \mathrm{~m}$ at maximal intensity, while for a $100-\mathrm{m}$ race, Seifert et al. [32] observed that the lowest performers (who seemed the most tired as they took $8.5 \mathrm{~s}$ more than the national swimmer group to complete the $100 \mathrm{~m}$ ) increased $\mathrm{IdC}$ from relative opposition $(-1.2 \%)$ to superposition coordination $(2.8 \%)$ between the third and last $25-\mathrm{m}$ lap.

These changes in arm coordination may be related to lower average hand speed, as Suito et al. [37] reported a decrease from 2.54 to $2.29 \mathrm{~m} \bullet \mathrm{s}-1$ between the 2 parts of the $100 \mathrm{~m}$. Toussaint et al. [40] showed that as fatigue developed during a maximal-intensity $100 \mathrm{~m}$, speed decreased by $12.4 \%$ and power by $23.5 \%$, hand speed dropped from $2.14 \mathrm{~m} \bullet \mathrm{s}-1$ at the first lap to $1.91 \mathrm{~m} \bullet \mathrm{s}-1$ at last lap and propelling efficiency from $81 \%$ at the first 3 laps to $76 \%$ at the last lap. Changes in stroke organization and notably IdC with fatigue were also observed for aerobic exercise [2][22]. During time limit to exhaustion tests swum at 95\%, 100\% and $110 \%$ of maximal aerobic speed, stroke length decreased while stroke rate increased over the test and arm coordination switched from catch-up mode (IdC -4\%) to opposition mode (IdC -1\%) [2]. Alberty et al. [2] hypothesized that fatigue development reduced the swimmers' capacity to generate a propulsive impulse per stroke. To compensate, the swimmers accorded greater time to propulsion per distance unit to maintain the overall propulsive impulse [2]. This was accomplished by increasing the IdC, which prompted Alberty et al. [2] to emphasize that these modifications in stroke organization did not guarantee better propulsion. In fact, as observed for sprint exercises, Monteil [22] showed a decrease in hand speed in the insweep phase from $2.5 \mathrm{~m} \bullet \mathrm{s}-1$ to $2 \mathrm{~m} \bullet \mathrm{s}-1$ through an exhausting $400-\mathrm{m}$ test in flume, and a decrease in the resultant force in the insweep and final outsweep phases. Our hypothesis may be tenable, given these findings about changes in arm coordination and propulsion during fatigue in sprint and aerobic exercise.

Second, it could be hypothesized that the sprint swimmers further modified their arm coordination because they usually focus on propulsion to overcome high active drag during sprint races. In fact, sprinters usually travel through a larger range of speeds in training and are accustomed to reaching higher maximal speeds than long-distance swimmers; thus, their arm coordination may appear more sensitive to environmental constraints (change in speed and high aquatic resistance). For example, Hue et al. [18] showed that triathletes maintained catch-up coordination when speed was increased from the 800-m race pace to the $100-\mathrm{m}$ race pace, possibly because they mostly reach lower speeds, train the aerobic pathway, and have lower propelling efficiency than swimmers. Conversely, swimmers increased their IdC with speed until reaching a relative opposition mode of coordination [18]. Toussaint [38] showed that during several 400-m trials swum at $0.7-1.35 \mathrm{~m} \bullet \mathrm{s}-1$, triathletes used a higher proportion of their power output than swimmers to overcome drag for an equal power input, supporting that triathletes have lower propelling efficiency. However, it is important to note that the higher coordination variability observed for sprinters mostly concerned high speeds, as only elite sprinters usually swim $\sim 2 \mathrm{~m} \bullet \mathrm{s}-1$ and have an effective superposition 
mode [31][33]. Indeed, Seifert et al. [31][33] observed a switch from catch-up to superposition coordination mode above the critical speed of $1.8 \mathrm{~m} \bullet \mathrm{s}-1$ and/or the critical stroke rate of $0.83 \mathrm{~Hz}$. In our progressive $6 \times 300 \mathrm{~m}$, the sprinters reached lower speeds than the long-distance swimmers; moreover, the speed of both groups remained below $1.6 \mathrm{~m} \bullet \mathrm{s}^{-1}$. Therefore, our second hypothesis that the changes in the sprinters' arm coordination could be related to greater motor flexibility remains limited. Indeed, McCabe and Sanders [21], who compared sprinters and long-distance swimmers during sprint (4 repeats of $25 \mathrm{~m}$ ) and aerobic $(400 \mathrm{~m})$ exercise, observed only temporal hand kinematic differences between the 2 groups during the sprint exercise, confirming that mostly high speeds impose great aquatic resistance that could induce changes in stroke organization.

\section{Conclusion}

In response to the interaction of environmental and task constraints (respectively, speed increases and instructions to swim $300 \mathrm{~m}$ for each set), the entire sample of swimmers increased $\mathrm{C}$, which corresponded to a decrease in Caero and an increase in Canaero. They also modified their stroking parameters by increasing stroke rate and decreasing both stroke length and inter-arm coordination (IdC) toward less of a catch-up mode. The progressive $6 \times 300 \mathrm{~m}$ test imposed aerobic sets that led to a greater increase in energy cost and Canaero for the sprinters than for the long-distance swimmers. Because sprinters spend more time training the anaerobic pathway, are accustomed to a greater speed range, and reach higher maximal speeds, this aerobic test caused them earlier muscular fatigue. They thus displayed lower performance (swim speed), lower efficiency (stroke index), higher propulsive continuities (higher IdC) and a greater change in arm coordination (increase in IdC) than the long-distance swimmers over the course of the test. Last, the volume of aerobic or anaerobic training and specialization in long-distance or sprint swimming have a great impact on the relationship of fatigue development and motor organization in swimmers. This suggests that coaches should consider arm coordination, as well as stroking parameters, when a specialization towards sprint or long-distance is chosen.

\section{References}

1 Alberty M, Sidney M, Huot-Marchand F, Hespel JM, Pelayo P. Intracyclic velocity variations and arm coordination during exhaustive exercise in front crawl stroke. Int J Sports Med 2005; 26: 471-475

2 Alberty M, Sidney M, Pelayo P, Toussaint HM. Stroking characteristics during time to exhaustion tests. Med Sci Sports Exerc 2009; 41: 637-644

3 Avalos M, Hellard P, Chatard JC. Modeling the training-performance relationship using a mixed model in elite swimmers. Med Sci Sports Exerc 2003; 35: 838-846

4 Barbosa T, Fernandes RJ, Keskinen KL, Vilas-Boas JP. The influence of stroke mechanics into energy cost of elite swimmers. Eur J Appl Physiol 2008; 103: 139-149

5 Barbosa T, Lima F, Portela A, Novais D, Machado L, Colaço P, Gonçalves P, Fernandes R, Keskinen K, Vilas-Boas JP. Relationships between energy cost, swimming velocity and speed fluctuation in competitive swimming strokes. Port J Sport Sci 2006; 6: 192-194

6 Capelli C, Pendergast DR, Termin B. Energetics of swimming at maximal speed in humans. Eur J Appl Physiol 1998; 78: 385-393

7 Cappaert JM, Bone M, Troup JP. Intensity and performance related differences in propelling and mechanical efficiencies. In: MacLaren D, Reilly T, Less A (eds) Swimming Science VI, England, London: E \& FN SPON; 1992: 49-52

8 Chatard JC, Collomp C, Maglischo E, Maglischo C. Swimming skill and stroking characteristics of front crawl swimmers. Int J Sports Med 1990; 11: 156-161

9 Chatard JC, Lavoie JM, Lacour JR. Analysis of determinants of swimming economy in front crawl. Eur J Appl Physiol 1990; 61: 88-92

10 Chollet D, Chalies S, Chatard JC. A new index of coordination for the crawl : description and usefulness. Int J Sports Med 2000; 21: 54-59 
11 Costill DL, Kovaleski J, Porter D, Kirwan J, Fielding R, King D. Energy expenditure during front crawl swimming: Predicting success in middle-distance events. Int J Sports Med 1985; 6: 266-270

12 Costill DL, Maglischo EW, Richardson AB. Swimming, Blackwell Science LTD,. 1992;

13 di Prampero PE. The energy cost of human locomotion on land and in water. Int J Sports Med 1986; 7: $55-72$

14 di Prampero PE, Feretti G. The energetics of anaerobic muscle metabolism: a reappraisal of older and recent concepts. Resp Physiol 1999; 118: 103-115

15 di Prampero PE, Salvadego D, Fusi S, Grassi B. A simple method for assessing energy cost of running incremental tests. J Appl Physiol 2009; 107: 1068-1075

16 Duffield R, Dawson B, Pinnington H, Wong P. Accuracy and reliability of a Cosmed K4b2 portable gas analyser. J Sci Med Sport 2004; 7: 11-22

17 Harriss DJ, Atkinson G. International Journal of Sports Medicine - Ethical Standards in Sport and Exercise Science Research. Int J Sports Med 2009; 30: 701-702

18 Hue O, Benavente H, Chollet D. Swimming skill in triathletes and swimmers using the index of coordination. J Hum Mov Studies 2003; 44: 107-120

19 Kaneko M, Fuchimoto T, Ito A, Toyooka J. Mechanical efficiency of sprinters and distance runners during constant speed running. In: Matsui H, Kobayashi K, (eds). Biomechanics VIII-B. Champaign, Illinois, Human Kinetics Publishers; 1983: 754-761

20 Lavoie JM, Montpetit RR. Applied physiology of swimming. Sport Med 1986; 3: 165-189

21 McCabe C, Sanders R. Are sprint and distance freestyle swimmers technically different? . http://www.coachesinfo.com/index.php?option=com content\&view=article \&id=10023: are-sprint-and-

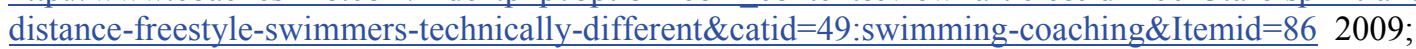

22 Monteil KM. Analyse biomécanique du nageur de crawl lors d'un test conduisant à épuisement: études des paramètres cinématiques, cinétiques et électromyographiques [Biomechanical analysis of a front crawl swimmer during an exhausting test: study of kinematic, kinetic and electromyographic parameters]. Unpublished PhD thesis, University of Claude Bernard, Lyon 1, France; 1992

23 Morais P, Vilas-Boas JP, Seifert L, Chollet D, Keskinen KL, Fernandes R. Relationship between energy cost and the index of coordination in front crawl - a pilot study. J Sports Sci 2008; 26 (Supplement 1) 11

24 Olbrecht J, Mader A, Heck H, Hollmann W. The importance of a calculation scheme to support the interpretation of lactate tests. In: MacLaren D, Reilly T, Less A, (eds) Swimming Science VI, England, London: E \& FN SPON; 1992: 243-249

25 Pyne DB, Boston T, Martin DT, Logan A. Evaluation of the Lactate Pro blood lactate analyser. Eur J Appl Physiol 2000; 82: 112-116

26 Rodriguez FA. Cardiorespiratory and metabolic field testing in swimming and water-polo: from physiological concepts to practical methods. In: Keskinen KL, Komi PV, Hollander AP, (eds). Biomechanics and Medicine in swimming VIII. Gummerus Printing, Jyvaskyla; 1999: 219-226

27 Rodríguez FA, Keskinen KL, Kusch M, Hoffmann U. Validity of a swimming snorkel for metabolic testing. Int J Sports Med 2008; 29: 120-128

28 Satkunskiene D, Schega L, Kunze K, Birzinyte K, Daly D. Coordination in arm movements during crawl stroke in elite swimmers with a locomotor disability. Hum Mov Sci 2005; 24: 54-65

29 Schnitzler C, Seifert L, Ernwein V, Chollet D. Arm coordination adaptations assessment in swimming. Int J Sports Med 2008; 29: 480-486 
30 Seifert L, Boulesteix L, Chollet D. Effect of gender on the adaptation of arm coordination in front crawl. Int J Sports Med 2004; 25: 217-223

31 Seifert L, Chollet D, Bardy B. Effect of swimming velocity on arm coordination in front crawl: a dynamical analysis. J Sports Sci 2004; 22: 651-660

32 Seifert L, Chollet D, Chatard JC. Changes in coordination and kinematics during a 100-m front crawl. Med Sci Sports Exerc 2007; 39: 1784-1793

33 Seifert L, Chollet D, Rouard A. Swimming constraints and arm coordination. Hum Mov Sci 2007; 26: $68-86$

34 Seifert L, Daly D, Burkett B, Chollet D. The profile of aquatic motor skills for able-bodied and swimmers with an impairment. In: LT Pelligrino (ed) Motor Skills: Development, Impairment and Therapy, Nova Science Publishers, Hauppauge, New York; 2009: 65-94

35 Shepard RJ, Godin G, Campbell R. Characteristics of sprint, medium and long-distance swimmers. Eur J Appl Physiol 1974; 32: 99-116

36 Sparrow WA, Newell KM. Metabolic energy expenditure and the regulation of movement economy. Psycho Bull Rev 1998; 5: 173-196

37 Suito H, IkegamiY, Nunome H, Sano S, Shinkai H, Tsujimoto N. The effect of fatigue on the underwater arm stroke motion in the 100-m front crawl. J Appl Biomech 2008; 24: 316-324

38 Toussaint HM. Differences in propelling efficiency between competitive and triathlon swimmers. Med Sci Sports Exerc 1990; 22: 409-415

39 Toussaint HM, Beelen A, Rodenburg A, Sargeant AJ, de Groot G, Hollander AP, van Ingen Schenau GJ. Propelling efficiency of front crawl swimming. J Appl Physiol 1988; 65: 2506-2512

40 Toussaint HM, Carol A, Kranenborg H, Truijens M. Effect of fatigue on stroking characteristics in an arms-only 100-m front-crawl race. Med Sci Sports Exerc 2006; 38: 1635-1642

41 Toussaint HM, Hollander AP. Energetics of competitive swimming. Sports Med 1994; 18: 384-405

42 Toussaint HM, Knops W, de Groot G, Hollander AP. The mechanical efficiency of front crawl swimming. Med Sci Sports Exerc 1990; 22: 402-408

43 Zamparo P, Bonifazi M, Faina M, Milan A, Sardella F, Schena F, Capelli C. Energy cost of swimming of elite long-distance swimmers. Eur J Appl Physiol 2005; 94: 697-704

44 Zamparo P, Pendergast DR, Termin B, Minetti AE. How fins affect the economy and efficiency of human swimming. J Exp Biol 2002; 205: 2665-2676 


\section{Figures and Table}

Fig. 1 Change in the stroking parameters along the 6 sets of $300 \mathrm{~m}$ for the sprint and long-distance swimmers; v: speed in $\mathrm{m} \cdot \mathrm{s}^{-1}$, SR: stroke rate in $\mathrm{Hz}$, SL: stroke length in $\mathrm{m} \cdot \mathrm{stroke}^{-1}$, SI: stroke index in $\mathrm{m}^{2} \cdot \mathrm{s}^{-1}$.

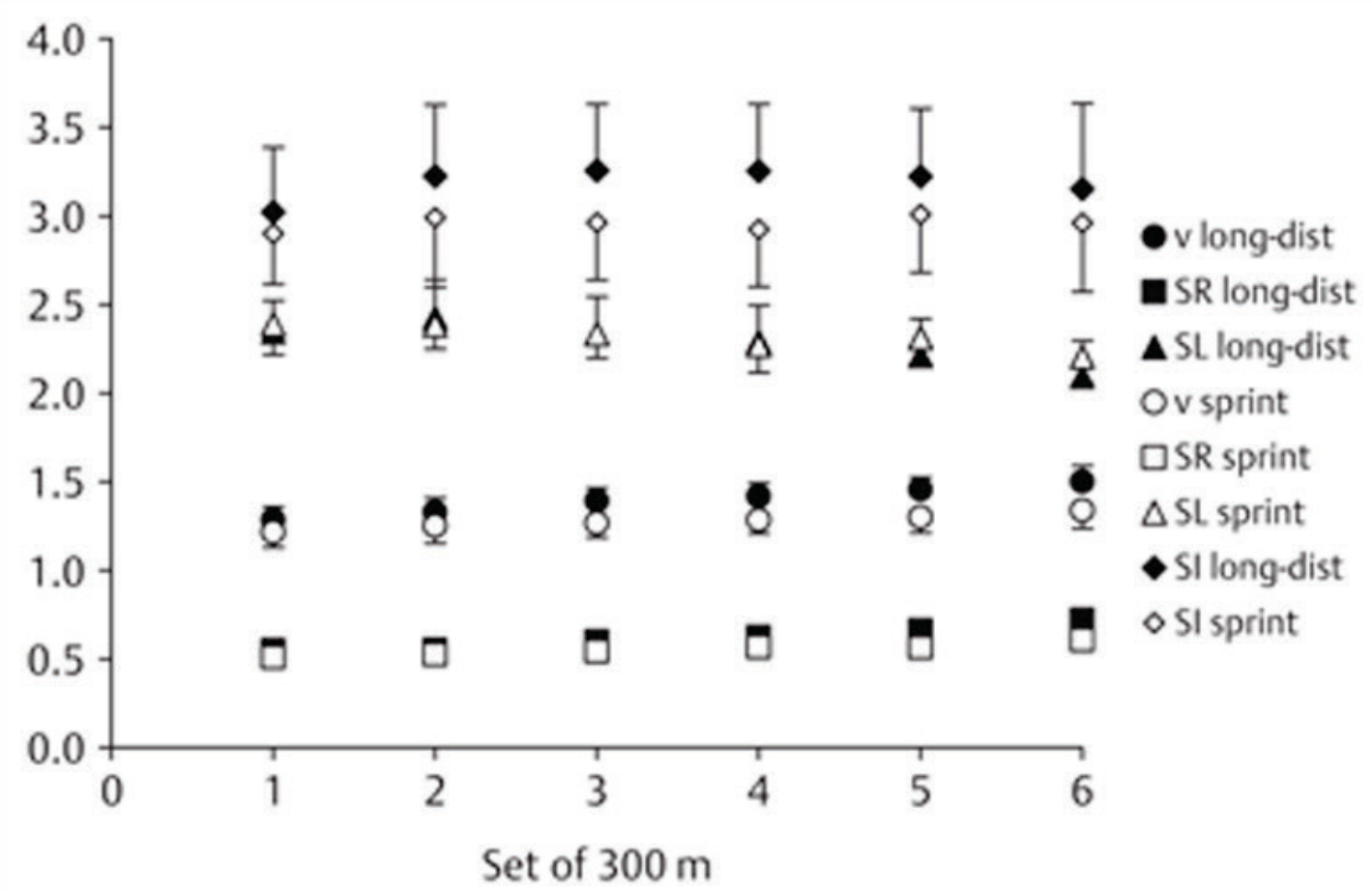


Fig. 2 Linear regression between the index of coordination (IdC) and energy cost $(C)$ for the sprint and long-distance swimmers.

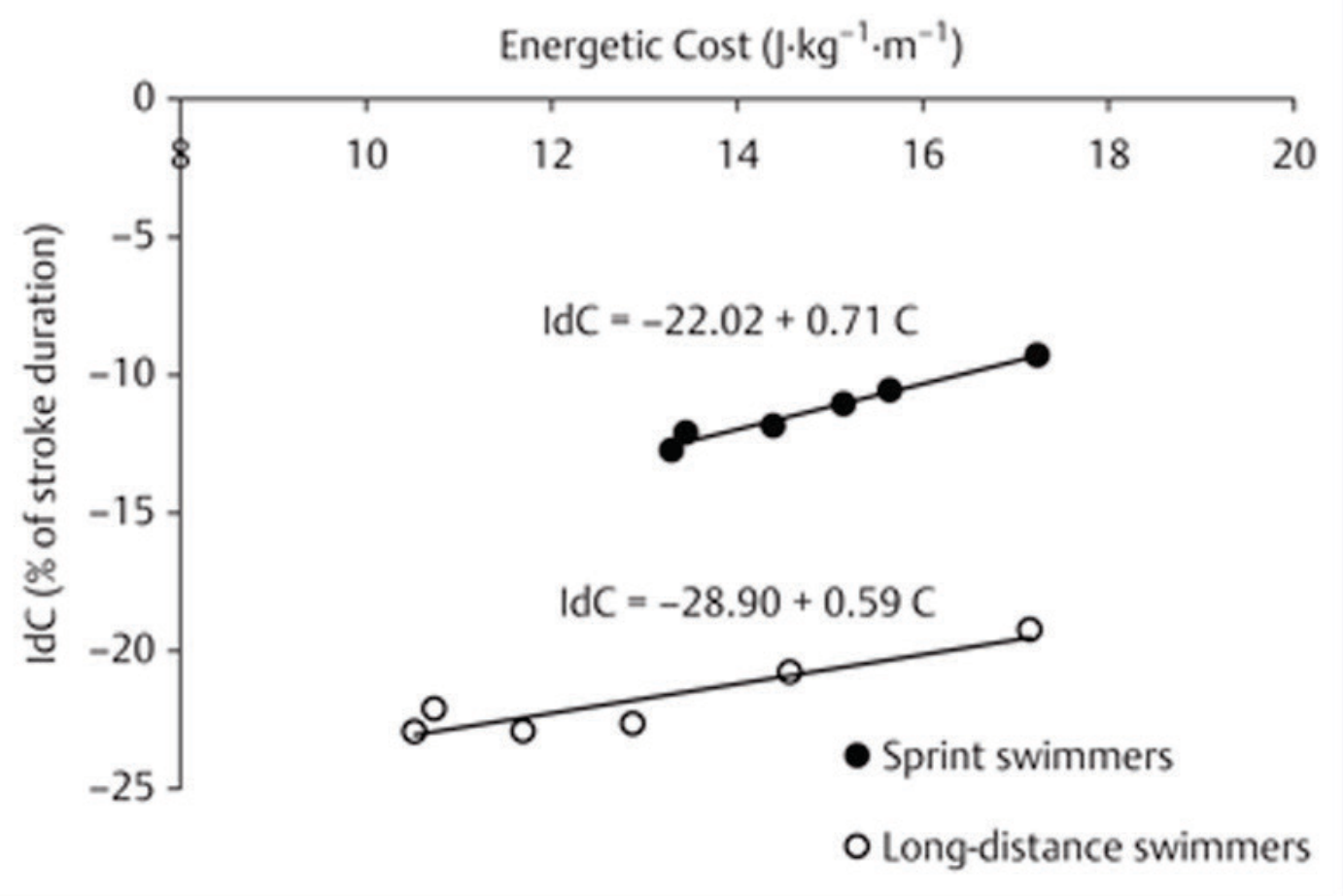


Fig. 3 Linear regression between index of coordination (IdC) and the aerobic component of energetic cost $\left(C_{\text {aero }}\right)$, and between IdC and the anaerobic component of energetic $\operatorname{cost}\left(C_{\text {anaero }}\right)$ for the sprint and longdistance swimmers.

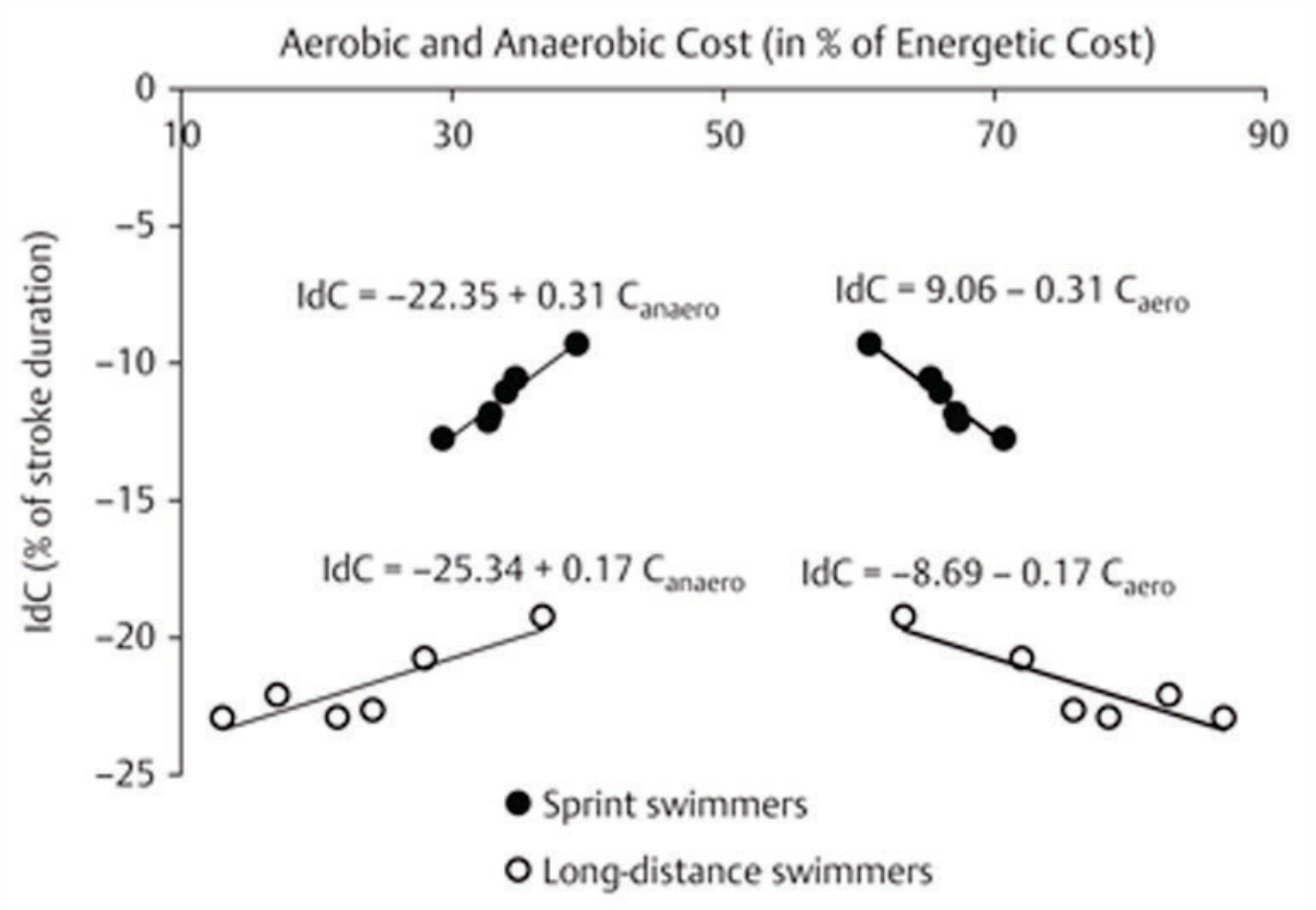


Table 1 Change in the energy cost $(C)$ and its aerobic $\left(C_{\text {aero }}\right)$ and anaerobic $\left(C_{\text {anaero }}\right)$ components along the 6 sets of $300 \mathrm{~m}$ increased in speed, for the sprint and long-distance swimmers.

\begin{tabular}{|c|c|c|c|c|c|c|c|c|c|c|c|c|}
\hline \multirow{3}{*}{$\begin{array}{l}\text { Set of } \\
300 \mathrm{~m}\end{array}$} & \multicolumn{6}{|c|}{ Long-distance swimmers } & \multicolumn{6}{|c|}{ Sprint swimmers } \\
\hline & \multicolumn{2}{|c|}{$C\left(J \cdot \mathrm{kg}^{-1} \cdot \mathrm{m}^{-1}\right)$} & \multicolumn{2}{|c|}{$C_{\text {aero }}(\%)$} & \multicolumn{2}{|c|}{$C_{\text {anaero }}(\%)$} & \multicolumn{2}{|c|}{$C\left(J \cdot \mathrm{kg}^{-1} \cdot \mathrm{m}^{-1}\right)$} & \multicolumn{2}{|c|}{$C_{\text {aero }}(\%)$} & \multicolumn{2}{|c|}{$C_{\text {anaero }}(\%)$} \\
\hline & Mean & $S D$ & Mean & $S D$ & Mean & $S D$ & Mean & $S D$ & Mean & $S D$ & Mean & $S D$ \\
\hline 1 & 10.5 & 2.3 & 86.9 & 6.1 & 13.1 & 6.1 & 13.3 & 2.4 & 70.7 & 8.2 & 29.3 & 8.2 \\
\hline 2 & 10.7 & 2.7 & 82.9 & 7.5 & 17.1 & 7.5 & 13.4 & 2.2 & 67.3 & 11.0 & 32.7 & 11.0 \\
\hline 3 & 11.7 & 2.7 & 78.5 & 6.9 & 21.6 & 6.9 & 14.4 & 1.9 & 67.2 & 9.9 & 32.9 & 9.9 \\
\hline 4 & 12.9 & 2.0 & 75.9 & 8.5 & 24.1 & 8.5 & 15.1 & 1.7 & 66.0 & 9.9 & 34.0 & 9.9 \\
\hline 5 & 14.6 & 2.2 & 72.0 & 9.0 & 28.0 & 9.0 & 15.6 & 1.6 & 65.3 & 8.6 & 34.7 & 8.6 \\
\hline 6 & 17.2 & 2.4 & 63.3 & 8.3 & 36.7 & 8.3 & 17.2 & 2.1 & 60.8 & 7.8 & 39.2 & 7.8 \\
\hline mean & 12.9 & 3.3 & 79.6 & 10.7 & 23.4 & 10.7 & 14.8 & 2.3 & 66.2 & 9.4 & 33.8 & 9.4 \\
\hline
\end{tabular}

